\title{
Vivaldi Antenna for UWB Sensor Networks
}

\author{
Jan Gamec ${ }^{1}$, Jan Schneider ${ }^{1}$, Maria Gamcova ${ }^{1}$ \\ ${ }^{1}$ Department of Electronics and Multimedia Comunications, Technical University of Kosice, \\ Park Komenskeho 13, 04120 Kosice, Slovak Republic \\ jan.gamec@tuke.sk
}

\begin{abstract}
In this paper, the Vivaldi antenna designed for ultra-wideband (UWB) sensor networks by exponential curves is presented. Manufactured antennas are used for through-wall measurements. The article describes the design and optimization of the antenna, antenna measurements using the network vector analyser and pulse radar. Measured results are compared with simulated results. The antenna is also compared with commercially manufactured antennas used in the above mentioned applications. The designed Vivaldi antenna is printed on ARLON 600 substrate and operates in the frequency band from $0.810 \mathrm{GHz}$ up to more than $12 \mathrm{GHz}$. Experimental results show good agreement with the simulated performance.
\end{abstract}

\section{Index Terms - Ultra wideband radar; Vivaldi antenna.}

\section{INTRODUCTION}

Nowadays, ultra-wideband radar holds great promise for a variety of applications. UWB radar was able to noninvasively detect movements of the heart wall [1], [10]. Motion sensing and imaging ultra-wideband (UWB) radar systems have strong potential for their use in medicine, impedance spectroscopy [2], ground penetrating radar systems [3], [9], through-wall measurements for emergency services [4], as well as in UWB sensor networks and other usable applications. A key element in any UWB radar system is one or more antennas, depending on the application [8].

In this paper, UWB antenna for through-wall measurements is described. The minimum ringing is one of the main requirements for the UWB antenna used in radar systems. Another requirement is covering the largest frequency bandwidth possible in which it operates. UWB device is defined as any device operating in absolute bandwidth greater than $500 \mathrm{MHz}$ or fractional bandwidth greater than 0.2 of central frequency [6]. Other required properties of the antenna for UWB radars include directivity, linear polarization, width of main lobe at least $90^{\circ}$, lowprofile, simple manufacturing and small dimensions while maintaining $\mathrm{f}_{\min }=1 \mathrm{GHz}$.

According to [5] the Vivaldi antenna was selected from the sets of UWB antennas. This antenna exhibits negligible dispersion and directional properties suitable for the planned

Manuscript received 15 December, 2015; accepted 28 May, 2016.

The research described in this paper was supported by the Scientific Grant Agency of the Ministry of Education of the Slovak Republic (ME SR) and of Slovak Academy of Sciences (SAS) under the contract No.1/0563/13 and Slovak Research and Development Agency under the contract No. APVV-0404-12. application, where negligible dispersion means a relatively constant group delay with varying frequency of operation. Figure 1 presents an application oriented classification of antennas.

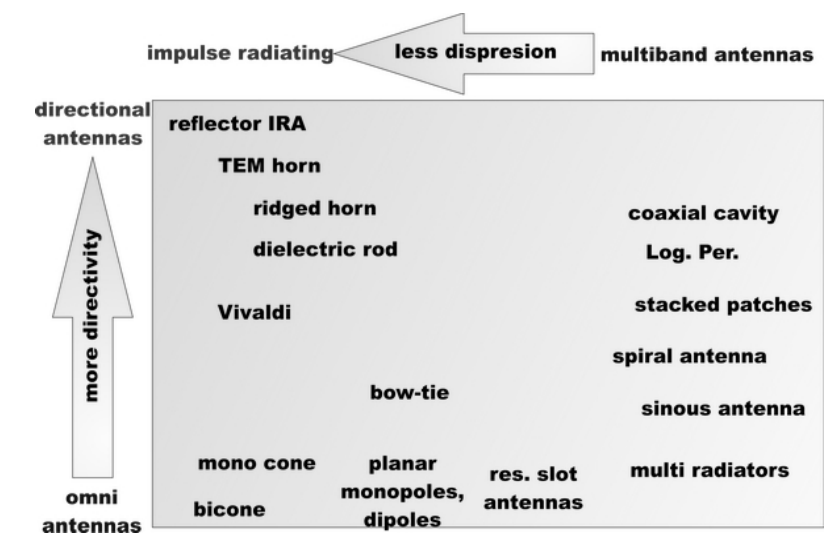

Fig. 1. Application oriented classification of antennas [5].

This article presents a planar antipodal Vivaldi antenna for UWB radars used in through-wall measurements. The following sections present the design of the antenna for UWB radars as well as simulated and measured results.

\section{ANTENNA DESIGN}

The configuration of the developed antenna is shown in Fig. 2. The antenna is an Antipodal Vivaldi antenna. The top layer is connected to the signal conductor of the feeding line and the bottom layer is connected to the ground plane of the feeding line.

The proposed antenna is printed on ARLON 600 substrate of dielectric constant $\varepsilon_{r}=6.15$, tangent loss $\tan \delta=0.003$ and thickness of $1.575 \mathrm{~mm}$. The dimensions of the substrate are $\mathrm{W}=128 \mathrm{~mm}$ and $\mathrm{L}=190 \mathrm{~mm}$. The width of the antenna $W$ is estimated by the lowest frequency of operation $f_{l}$ and by dielectric constant of the substrate $\varepsilon_{r}$. The width $(W)$ is calculated using the following equation [7]

$$
W=\frac{c}{f_{1}} \sqrt{\frac{2}{\varepsilon_{r}+1}},
$$

where $c$ is the speed of light in free space, $f_{1}$ is the lowest operating frequency and $\varepsilon_{r}$ is permittivity.

The appearance of inner side of the antenna arms (darker curve in Fig. 2) is based on the principle of exponential curves. Curve equation has the form (CST MWS uses analytical expressions of the following equation) 


$$
l=\frac{L_{2}}{\sqrt[A]{W_{3}}} \times w^{\frac{1}{A}}
$$

where the variable $A$ is the effect on the shape of exponential curve, the variables $L_{2}$ and $W_{3}$ adjust the exponential of $l$ and $w$ axes and $w$ is width of the exponential curve.

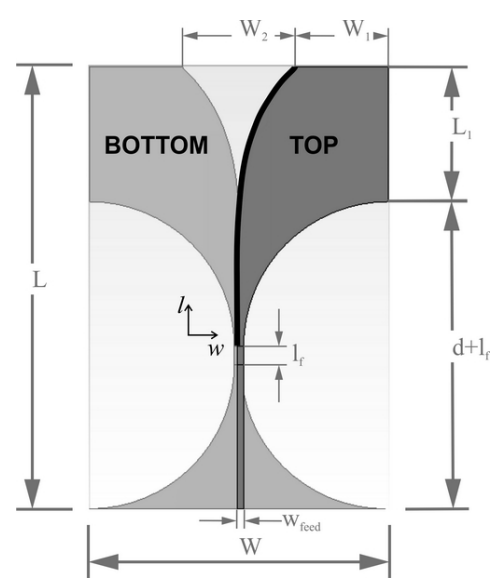

Fig. 2. Antipodal Vivaldi antenna with geometry parameters.

The radiating elements of the antenna are formed by connecting curves such as lines (W1, L1 etc.) and quarter circles (both sides of the line lf). Curves are designed in the same way using the analytical curves. All statements curves of the antenna have their own variables. Variables were tuned and optimized in order to obtain the required properties of the antenna.

\section{Simulated AND Measured Results}

\section{A. Measurement of Antennas in Anechoic Chamber}

This section presents simulation and experimental results of the proposed antenna. The simulation of the antenna was performed using full wave electromagnetic simulator CST Microwave Studio (CST MWS). The antenna was analyzed by the time domain solver. Also, CST MWS was used for tuning and optimization of the antenna parameters. For optimization of the final parameters 'trust region framework algorithm' algorithm was used.

The greatest impact on the return loss and radiation pattern of the proposed antenna have the following parameters:

- curvature of the exponential curve of the arm,

- the mutual distance between the arms (W2),

- and the length of the arms.

According to Fig. 2, the optimized dimensions and final shape of the designed antenna with port of the antenna are as follows: $\mathrm{W}_{1}=39.55 \mathrm{~mm}, \mathrm{~W}_{2}=48.94 \mathrm{~mm}, \mathrm{~L}_{1}=58 \mathrm{~mm}, \mathrm{l}_{\mathrm{f}}=$ $8 \mathrm{~mm}, \mathrm{~d}=124 \mathrm{~mm}$. The width of the microstrip feed line $\mathrm{W}_{\text {feed }}=2.75 \mathrm{~mm}$ is calculated and matched to the characteristic impedance $Z_{0}=50 \Omega$. The port was included in the simulation model.

The simulation results obtained by CST MWS are given in Fig. 3-Fig. 6 for direct comparison between simulation and experimental measurement. The frequency responses of the magnitude of the return loss at the input of the simulated antenna and the measured antenna are shown in Fig. 3. The far field radiation pattern and return loss of the considered antenna were measured by a network vector analyser in the rectangular anechoic chamber operating in the range from tens of $\mathrm{MHz}$ to $20 \mathrm{GHz}$.

As shown in Fig. 3, the return loss of the simulated and measured antenna is adjusted to $-10 \mathrm{~dB}$ over the frequency band from $0.810 \mathrm{GHz}$ to more than $12 \mathrm{GHz}$ with two small acceptable variations at the beginning to satisfy the requirements of the UWB technology. Also, Fig. 3 shows that the simulation and measurement results are in good agreement.

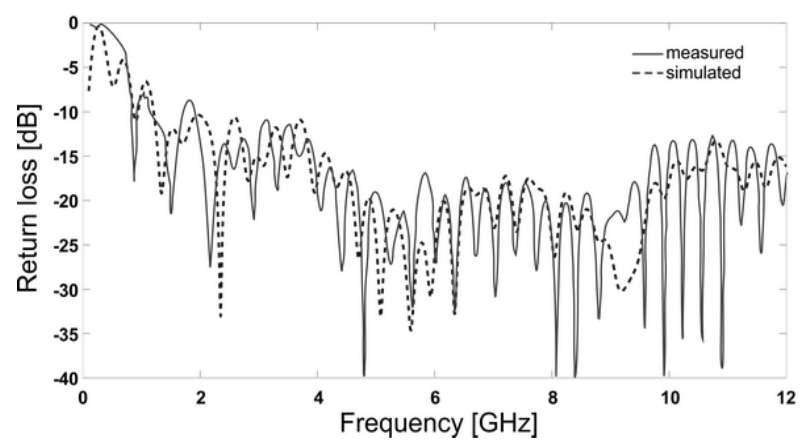

Fig. 3. Return losses of measured and simulated antenna.

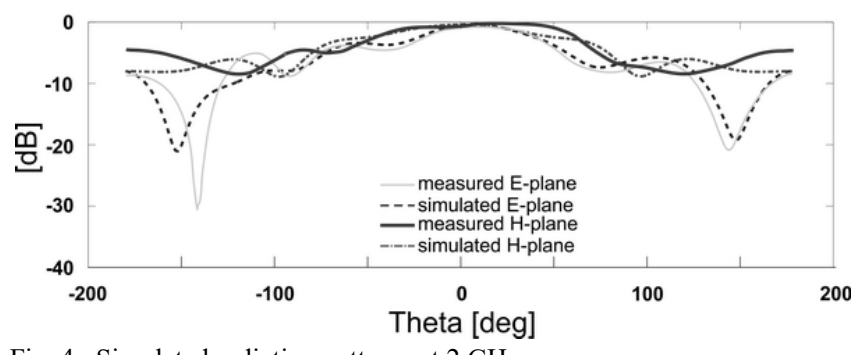

Fig. 4. Simulated radiation patterns at $2 \mathrm{GHz}$.

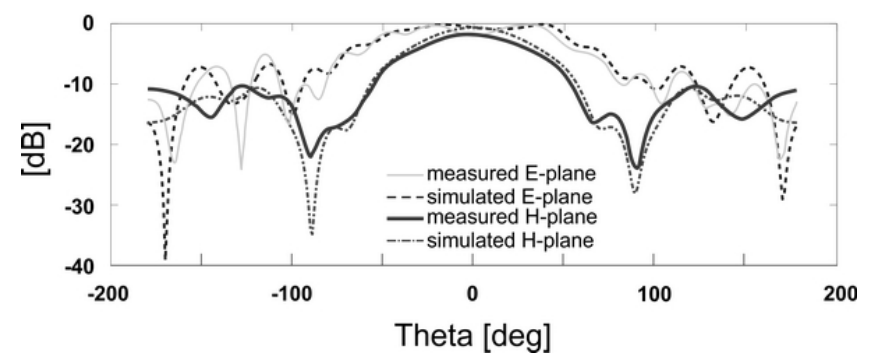

Fig. 5. Simulated radiation patterns at $4 \mathrm{GHz}$.

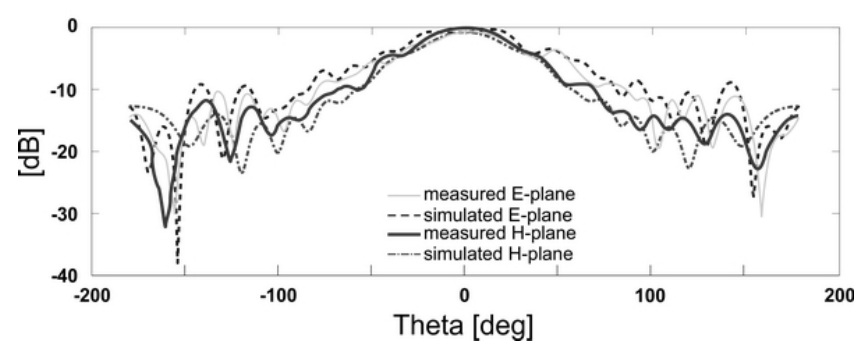

Fig. 6. Simulated radiation patterns at $6 \mathrm{GHz}$.

The simulated and measured radiation pattern is displayed in Fig. 4 to Fig. 6. The E-plane and H-plane radiation patterns are given at three different frequencies $2 \mathrm{GHz}$, $4 \mathrm{GHz}$ and $6 \mathrm{GHz}$. The comparison between the simulated radiation pattern and the measured radiation pattern of the proposed antenna shows good agreement between the two results. 


\section{B. Measurement Antennas with UWB Pulse Radar}

For time domain measurements, pulse UWB radar was used. It transmits a narrow pulse with peak amplitude $-\mathrm{U}<$ $30 \mathrm{~V} / 50 \Omega$ and width of the pulse $-\mathrm{t}<50$ ps. The principle diagram of measurements with the pulse UWB radar is shown in Fig. 7.

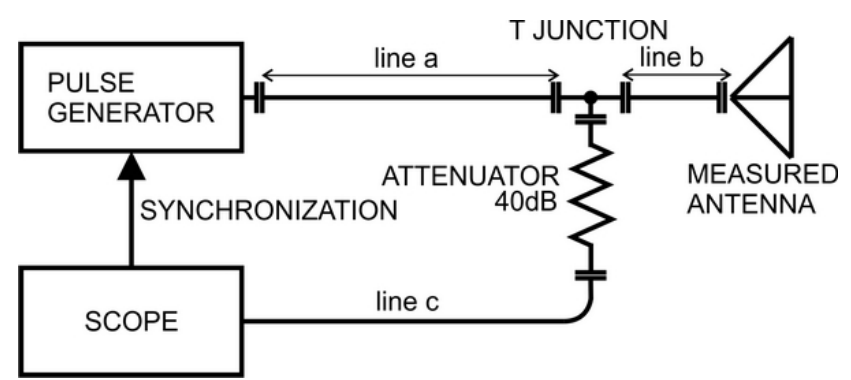

Fig. 7. Block diagram of measurement with the pulse UWB radar.

The reflected signals were received from the antenna after adjusting the observation window of the scope according to the lengths of lines $a$ and $b$. The shape and reference position of the excitation signal entering the antenna were obtained by replacing the antenna with the calibration probe of OPEN type.

The scale of the exciting pulse in the obtained results (in figures) of the measurement was decreased (y scale of impulse was reduced). The pulse characteristics of the proposed Vivaldi antenna are shown in Fig. 9 to Fig. 13.

The designed Vivaldi antenna together with the commercial wideband antennas are measured as described above.

The measured results in the time domain of the designed antenna were compared with the other wideband antennas (Fig. 8) and are given in Fig. 9- Fig. 13 as follows:

- small bow-tie: GEOZONDAS AU - 1.0G4.5GR

(1.0 GHz-4.5 GHz),

- large bow-tie: GEOZONDAS AU - 0.8G2.4G-B

(0.8 GHz-2.4 GHz),

- Double ridged waveguide horn: RFspin DRH10 (0.74 GHz-10.5 GHz),

- Quad Ridged Horn Antenna: RFspin QRH400 (0.4 GHz-6 GHz) (Port A and Port B).

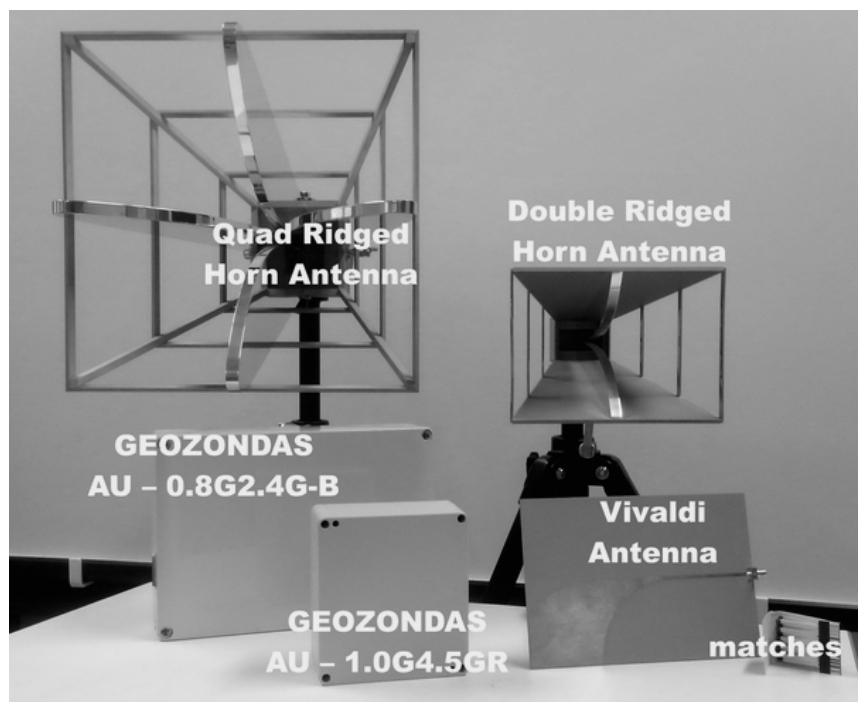

Fig. 8. Compared antennas.

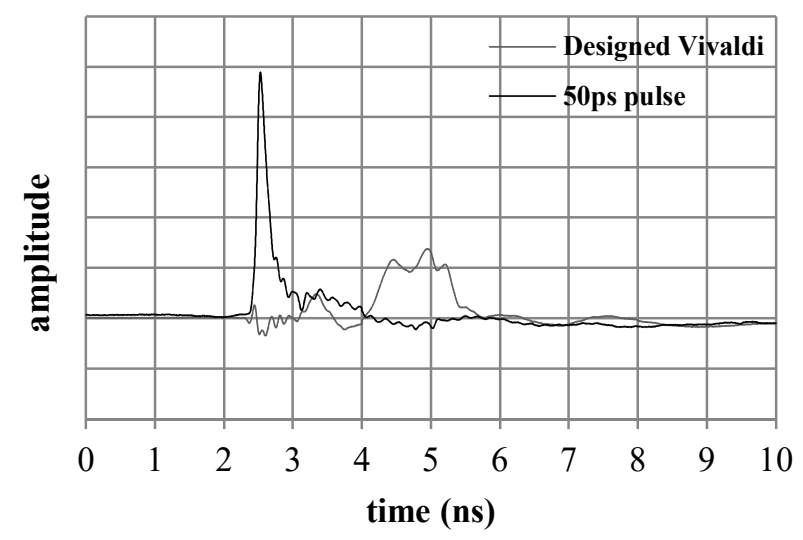

Fig. 9. Reflected response of the manufactured Vivaldi antenna on exciting pulse.

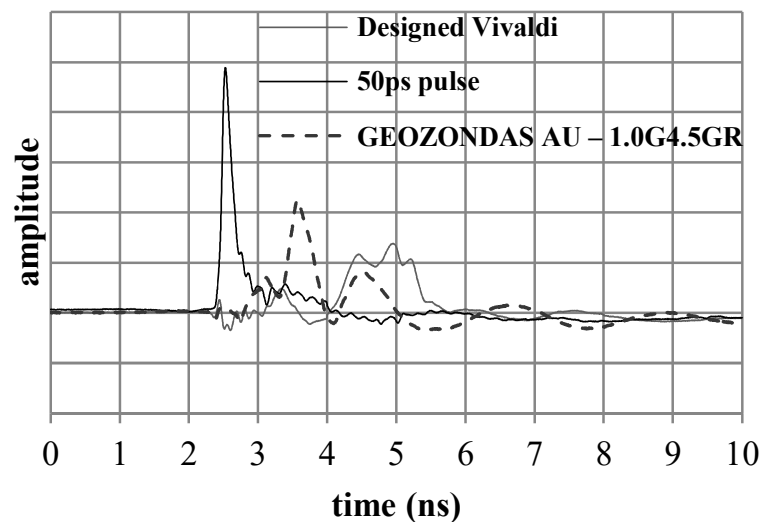

Fig. 10. Reflected response of the manufactured Vivaldi antenna compared with GEOZONDAS AU - 1.0G4.5GR.

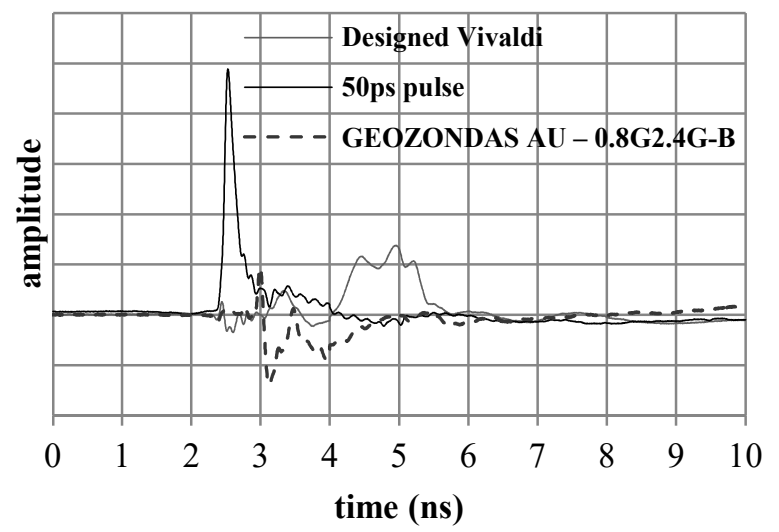

Fig. 11. Reflected response of the manufactured Vivaldi antenna compared with GEOZONDAS AU - 0.8G2.4G-B.

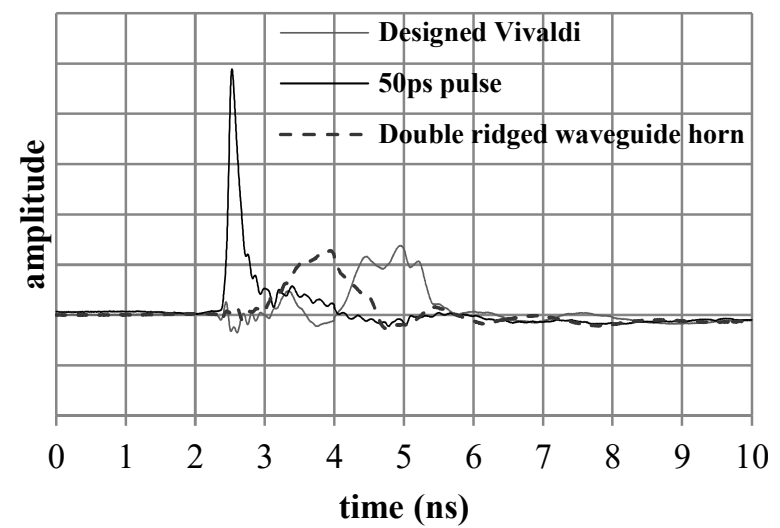

Fig. 12. Reflected response of the manufactured Vivaldi antenna compared with Double ridged waveguide horn.

The reflected impulse responses (Fig. 9-Fig. 13) of the 
proposed Vivaldi antenna have satisfactory frequency character in the wide bandwidth applications. Positive feedback from measurements in the time domain is that antenna has a very low ringing in comparison with commercially available antennas. The result presented above predestinates the manufactured antenna for applications sensitive to changes in group delay receiving and transmitting signal - UWB radar systems.

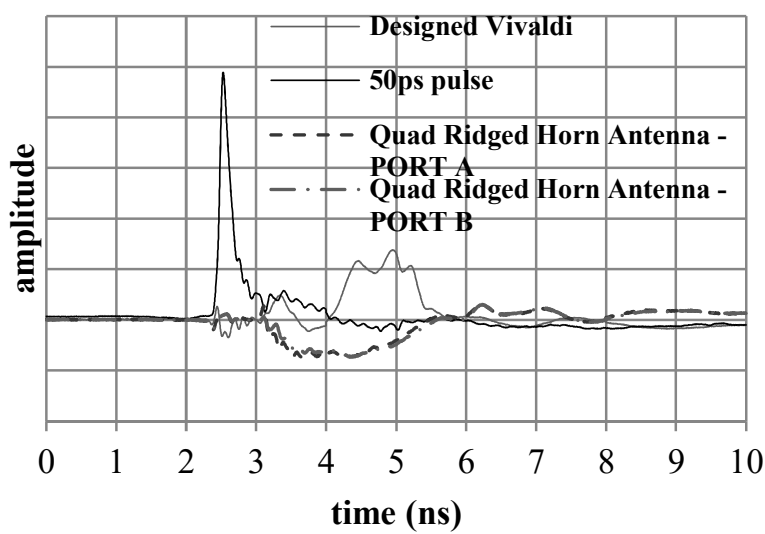

Fig. 13. Reflected response of the manufactured Vivaldi antenna compared with Quad Ridged Horn Antenna.

Except measurements using a network vector analyser, the return losses of the antenna were calculated from the impulse response (Fig. 9) of the UWB radar. The return loss of the manufactured antenna was obtained by following equation

$$
\begin{gathered}
s_{11}(n \Delta \omega)=\mathscr{F}\left(v_{\mathrm{A}}(n \Delta t)-v_{\mathrm{L}}(n \Delta t)\right) . / \\
\mathcal{F}\left(-v_{\mathrm{S}}(n \Delta t)-v_{\mathrm{L}}(n \Delta t)\right)
\end{gathered}
$$

where $s_{11}(n \Delta \omega)$ is reflection coefficient of the antenna, $\mathcal{F}$ is discrete Fourier transform operator, "./" is dot dividing, $v_{\mathrm{A}}(n \Delta t)$ is the impulse response of the antenna, $v_{\mathrm{L}}(n \Delta t)$ is the impulse response of termination of the type LOAD and $v_{\mathrm{S}}(n \Delta t)$ is the impulse response of termination of the type SHORT.

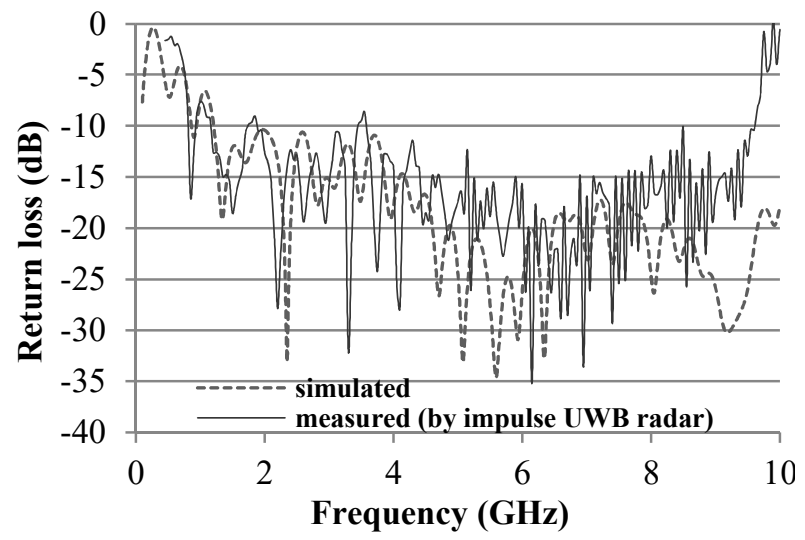

Fig. 14. Simulated and measured (by UWB radar) return loss of the antenna.

Expression " $v_{\mathrm{A}}(n \Delta t)-v_{\mathrm{L}}(n \Delta t)$ " represents the reflected signal without the influence of the lines and of the entire system. Expression " $-v_{\mathrm{S}}(n \Delta t)-v_{\mathrm{L}}(n \Delta t)$ " represents the incident signal without the influence of the lines and of the entire system too. If the Fourier transforms of the incident and the reflected signal are put into ratio, then reflection coefficient as function of frequency steps is obtained. Return loss $R L_{d B}$ in $\mathrm{dB}$ scale was calculated by the following equation

$$
R L_{d B}=20 \log _{10}\left(s_{11}(n \Delta \omega)\right)
$$

Simulated and measured (with UWB pulse radar) return loss of the manufactured antenna is shown in Fig. 14.

From the results obtained, it is obvious that the results of measurements in the anechoic chamber and those from impulse responses as well as simulation results show good agreement up to $5 \mathrm{GHz}$. The diversity of return loss over the mentioned frequency $5 \mathrm{GHz}$ is observed. This gradual increase of diversity may be caused by noise and hardware bandwidth limitations of the pulse UWB radar.

\section{CONCLUSIONS}

The suitable antipodal Vivaldi antenna for the UWB radars/sensor networks was presented in this paper. The antenna was designed, optimized and simulated in the software CST Microwave Studio. Figure 15 shows the manufactured antenna.

The frequency band of the antenna is from $0.81 \mathrm{GHz}$ to $12 \mathrm{GHz}$ with average gain $6.32 \mathrm{dBi}$ and fractional bandwidth $\mathrm{BW}=163 \%$. The return loss of the antenna between 0.81 $\mathrm{GHz}$ to $2 \mathrm{GHz}$ varies around the value $-10 \mathrm{~dB}$ (Fig. 3). This small variation is acceptable for UWB radars requirements and through-wall measurements with the above mentioned radars. All the measurement results confirmed good agreement with the simulated results.

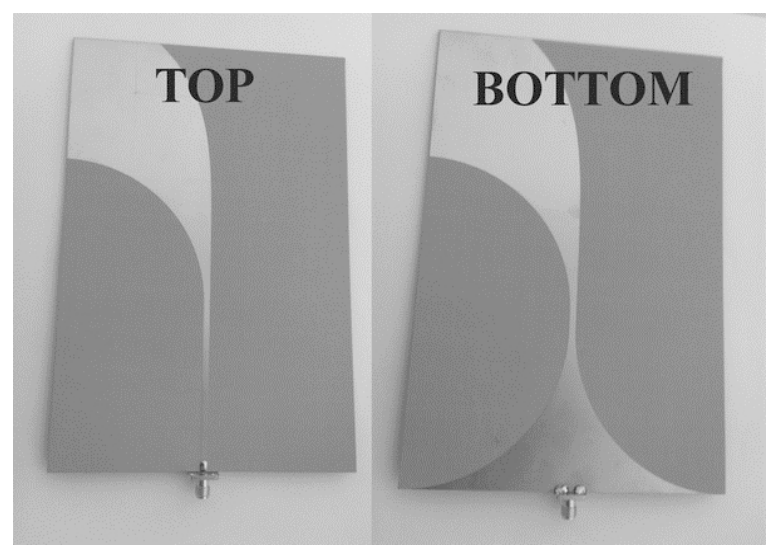

Fig. 15. Manufactured antenna.

Wide bandwidth of the designed antenna, a relatively small size along with the low cost of its production provide the properties suitable for the use in through-wall measurements with the UWB radars and can be replaced with commercial antennas presented in this paper.

\section{REFERENCES}

[1] E. M. Staderini, "UWB radars in medicine", IEEE Aerospace and Electronic Systems Magazine, vol. 17, no. 1, pp. 13-18, 2002. [Online]. Available: http://dx.doi.org/10.1109/62.978359

[2] M. Ziga, P. Galajda, S. Slovak, M. Kmec, "Determination of the quality of frying oil based on UWB impedance spectrometer", 16th Int. Radar Symposium (IRS 2015), Dresden, 2015, pp. 955-960. [Online]. Available: http://dx.doi.org/10.1109/IRS.2015.7226378

[3] D. H. N. Marpaung, Yilong Lu, "A comparative study of migration algorithms for UWB GPR images in SISO-SAR and MIMO-array 
configurations", 15th Int. Radar Symposium (IRS 2014), Gdansk, 2014, pp. 1-4. [Online]. Available: http://dx.doi.org/10.1109/ IRS.2014.6869192

[4] D. Novak, R. Zetik, D. Kocur, "Defective localization of target in UWB radar applications", 24th Int. Conf. Radioelektronika (RADIOELEKTRONIKA 2014), Bratislava, 2014, pp. 1-4. [Online]. Available: http://dx.doi.org/10.1109/Radioelek.2014.6828432

[5] X. Li, W. Wiesbeck, T. Zwick, "Design considerations for UWB antennas", 7th European Conf. Antennas and Propagation (EuCAP 2013), Gothenburg, 2013, pp. 558-561.

[6] D. Kocur et al., "Short-range UWB radar: Surveillance robot equipment of the future", IEEE Int. Conf. Systems, Man and Cybernetics (SMC 2014), San Diego, CA, 2014, pp. 3767-3772. [Online]. Available: http://dx.doi.org/10.1109/SMC.2014.6974517

[7] A. M. Abbosh, H. K. Kan, M. E. Bialkowski, "Design of compact directive ultra wideband antipodal antenna", Microw. Opt. Technol. Lett., vol. 48, pp. 2448-2450. [Online]. Available: http://dx.doi.org/ $10.1002 /$ mop. 21955

[8] J. Schneider, J. Gamec, "Overview of UWB low-profile planar antennas", Acta Electrotechnica et Informatica, vol. 14, no. 2, pp. 55-59, 2014. [Online]. Available: http://dx.doi.org/10.15546/ aeei-2014-0019.

[9] J. Labun, M. Sotak, P. Kurdel, "Technical note innovative technique of using the radar altimeter for prediction of terrain collision threats", The Journal of the American Helicopter Society, vol. 57, no. 4, 2012. [Online]. Available: http://dx.doi.org/10.4050/JAHS.57.045002

[10] C. N. Paulson et al., "Ultra-wideband radar methods and techniques of medical sensing and imaging", Optics East 2005, International Society for Optics and Photonics, 2005. [Online]. Available: http://dx.doi.org/doi: 10.1117/12.630004 